\title{
A Modified Artificial Bee Colony for the Non- Smooth Dynamic Economic/Environmental Dispatch
}

\author{
I. Marouani \\ National School of Engineering \\ of Sfax (ENIS), University of \\ Sfax, Sfax, Tunisia \\ ismailmarouani@yahoo.fr
}

\author{
A. Boudjemline \\ University of Hail, \\ Hail, Saudi Arabia \\ a.boudjemline@uoh.edu.sa
}

\author{
T. Guesmi \\ College of Engineering, \\ University of Hail, \\ Hail, Saudi Arabia \\ tawfiq.guesmi@gmail.com
}

\author{
H. H. Abdallah \\ National School of Engineering \\ of Sfax (ENIS), University of \\ Sfax, Sfax, Tunisia \\ Hsan.Hadj@enis.rnu.tn
}

\begin{abstract}
This paper presents an improved artificial bee colony (ABC) technique for solving the dynamic economic emission dispatch (DEED) problem. Ramp rate limits, valve-point loading effects and prohibited operating zones (POZs) have been considered. The proposed technique integrates the grenade explosion method and Cauchy operator in the original $\mathrm{ABC}$ algorithm, to avoid random search mechanism. However, the DEED is a multi-objective optimization problem with two conflicting criteria which need to be minimized simultaneously. Thus, it is recommended to provide the best solution for the decision-makers. Shannon's entropy-based method is used for the first time within the context of the on-line planning of generator outputs to extract the best compromise solution among the Pareto set. The robustness of the proposed technique is verified on sixunit and ten-unit system tests. Results proved that the proposed algorithm gives better optimum solutions in comparison with more than ten metaheuristic techniques.
\end{abstract}

Keywords-evolutionary computation; power generation dispatch; optimal scheduling; decision making; cost function

\section{INTRODUCTION}

Emission dispatch aims at minimizing emission of harmful gases, caused by fossil-fueled thermal units, such as $\mathrm{CO}, \mathrm{CO}_{2}$, $\mathrm{NO}_{\mathrm{x}}$ and $\mathrm{SO}_{2}$ [1-2]. The combination of the above problems is called the economic emission dispatch (EED) problem. However, due to the dynamic nature of today's network loads, it is required to schedule the thermal unit outputs in real time according to the variation of power demands during a certain time period [3]. To solve this modified EED problem, known as dynamic economic emission dispatch (DEED), several mathematical formulations have been suggested [3-6]. Usually the DEED problem is considered as a dynamic optimization problem having the same objectives as the EED over a timeperiod of one day, subdivided into definite time intervals of one hour with respect to the constraints imposed by the generator ramp-rate limits (RRL) [3]. Therefore, the operational decision at an hour may be influenced by the one taken at a previous hour. Other constraints such as prohibited operating zones (POZ) and valve-point loading effects (VPLE) have been considered [7-8]. However, incorporating VPLE in the fuel cost function introduces ripples in the latter and the problem will be with multiple minima. On the other hand, $\mathrm{POZ}$ constraints due to physical operation limitation like vibrations in the shaft bearing [9] create discontinuities in the objective functions. Therefore, the DEED becomes a highly nonlinear problem with non-convex and discontinuous fitness functions.

Classical methods like, dynamic programming [10] and linear programming [11], have been used to solve the static EED. However, these techniques are iterative and require an initialization step, which can cause the convergence of the search process into local optima. Moreover, they may fail to solve the dynamic case including the above constraints. Recently, metaheuristic search algorithms have demonstrated good performance and high efficiency when applied to complex optimization problems. These optimization procedures are classified into various groups in terms of the optimization methodology. Swarm intelligence-based evolutionary algorithms are the most used algorithms. Among metaheuristic-based optimization techniques, genetic algorithm [12], particle swarm optimization [13], simulated annealing [14], artificial bee colony (ABC) [7], tabu search [15], differential evolution [4] and bacterial foraging [5] have been suggested for solving the EED problem. Despite the fact that these techniques have been proven to have a clear edge over traditional methods, they have been criticized [16] because their efficiencies are sensitive to the form of problem constraints and number of units. Most of the above-mentioned works have concentrated only on the static EED problem. Only a few considered the multi-objective DEED problem. In addition, RRL and POZ constraints were not considered during the transition from the last hour of the current day to the first hour of the next day.

$\mathrm{ABC}$ algorithms attracted much attention for EED problems [9]. ABC algorithm [17] simulates the foraging behavior of a real bee colony for maximizing the nectar amount stocked in the hive. Compared to several population-based techniques like, PSO and GA, the $\mathrm{ABC}$ algorithm is simple in concept with a few setting parameters, easy for combination with other optimization approaches and more effective. Unfortunately, like other evolutionary algorithms, the $\mathrm{ABC}$ method has also been criticized for its poor convergence rate and premature convergence due to the unbalanced exploration-exploitation processes [16]. Exploration corresponds to the capability to avoid convergence toward local optima by expanding the search into new areas, while exploitation is the capability to 
search the space to find interesting new solutions. Thus, many improved techniques have been proposed to further enhance its performance [16-17]. In order to increase $A B C$ 's exploitation ability, the classical employed bee and onlooker bees' phases have been changed by incorporating the so-called grenade explosion method (GEM) [18]. The GEM is an optimization method proposed in [19] and, as the name suggests, it imitates the mechanism of a grenade explosion. The effectiveness of this modified version of $\mathrm{ABC}$, symbolized by $\mathrm{GABC}$, was verified on a set of standard reference functions. In [16], the Cauchy operator is embedded into the scout bees' phase of the $\mathrm{ABC}$ in order to increase the exploration ability by generating a larger set of solutions instead of one random solution for each scout bee.

A new method exploiting the advantages of GEM and Cauchy operator is proposed in the present work in order to solve the DEED problem with respect to all the abovementioned constraints. This optimization, symbolized by GCABC, integrates the GEM and Cauchy operator into the $\mathrm{ABC}$ technique. In addition, a new decision-making method based on Shannon's entropy, called extended entropy-weighted reference (EEWR) approach, is developed and incorporated into the GCABC algorithm to select the most suitable solution among all non-dominated solutions provided by the optimization algorithm. Unlike other techniques like those based on graph theory [20] and Z-transformation [21], the EEWR is characterized by uncomplicated mathematics [22]. The main contributions of the current work are:

- A new optimization technique, called GCABC, for scheduling power production of thermal units according to the expected load variations is proposed. To the best of our knowledge, the present work is the first attempt to solve the EED problem by the use of GCABC algorithm. In addition, a modified EWR-based technique, called extended entropyweighted reference (EEWR), is proposed for decision making. This technique has not been used in any field of power systems.

- All aforementioned constraints are considered simultaneously in the DEED problem.

- The RRL constraints are taken into account during the transition from the last hour of the one day to the first hour of the next day.

\section{PROBLEM FORMULATION}

The DEED problem is considered as a multi-objective optimization problem (MOP). It aims to minimize simultaneously total fuel cost and total emission by finding the power production of thermal power plants according to the predicted load demands. The resolution of the DEED problem can be accomplished by solving the static EED (SEED) problem over a certain period of time subdivided into smaller time intervals. DEED problem objectives and constraints are described below.

\section{A. Objective Functions}

Thermal units with multi-steam admission valves that work sequentially to cover the demand, involves higher order nonlinearity to total fuel cost due to VPLE, as illustrated in Figure 1. Unfortunately, neglecting the VPLE, which is required when using classical methods, causes some inaccuracy in the solution of the DEED problem. Taking into account VPLE constraints, a sinusoidal form is included in the total non-smooth cost function expressed in $\$ / \mathrm{h}$, as given in (1). The second objective corresponding to the total emission in ton $/ \mathrm{h}$ is described by (2):

$$
\begin{aligned}
& C_{T}=\sum_{t=1}^{T} \sum_{i=1}^{N} a_{i}+b_{i} P_{i}^{t}+c_{i}\left(P_{i}^{t}\right)^{2}+\left|d_{i} \sin \left\{e_{i}\left(P_{i}^{\min }-P_{i}^{t}\right)\right\}\right| \\
& E_{T}=\sum_{t=1}^{T} \sum_{i=1}^{N} \alpha_{i}+\beta_{i} P_{i}^{t}+\gamma_{i}\left(P_{i}^{t}\right)^{2}+\eta_{i} \exp \left(\lambda_{i} P_{i}^{t}\right)
\end{aligned}
$$

where, $a_{i}, b_{i}, c_{i}, d_{i}$ and $e_{i}$ are the cost coefficients of the $i$-th unit. While, $\alpha_{i}, \beta_{i}, \gamma_{i}, \eta_{i}$ and $\lambda_{i}$ are the emission coefficients. $P_{i}^{t}$ is the output power in MW at the $t$-th interval. $T$ is the number of hours. In this study, $T=24$.

The bi-objective DEED problem is converted into a monoobjective optimization problem in [23]. In this study, the price penalty factor (PPF)-based method is adopted. Thus the combined economic-emission objective function $F_{T}$ can be described by (3):

$$
F_{T}=\mu C_{T}+(1-\mu) \lambda E_{T}
$$

where, $\mu=\operatorname{rand}(0,1)$. For each generated value of $\mu$, the function $F_{T}$ is minimized to obtain the optimum solution that can be a candidate solution to be in the Pareto front. The parameter $\lambda$ is the average of the PPF of all thermal units. As shown in (4), the PPF of the $i$-th unit is the ratio between its fuel cost, $C_{i_{\max }}$, and its emission, $E_{i_{\max }}$, for maximum generation capacity.

$$
P P F_{i}=\frac{C_{i_{\max }}}{E_{i_{\max }}}
$$

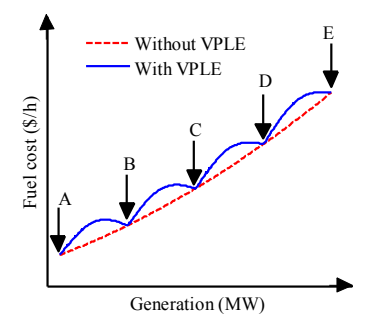

Fig. 1. Fuel cost function with five valves (A, B, C, D, E)

\section{B. Problem Constraints}

The DEED problem is solved by minimizing the function $F_{T}$ defined in (3) with respect to the following constraints:

- Generation capacity

The real power output of each unit $i$ should be within its minimum and maximum limits $P_{i}^{\min }$ and $P_{i}^{\max }$ : 


$$
P_{i}^{\min } \leq P_{i}^{t} \leq P_{i}^{\max }, i=1, \ldots, N
$$

- Power balance constraints

At each time period $t$, the total power generation must cover the total demand power $P_{D}^{t}$ plus the total transmission losses $P_{L}^{t}$. Thus, the power balance constraints can be described by:

$$
\sum_{i=1}^{N} P_{i}^{t}-P_{D}^{t}-P_{L}^{t}=0, t=1, \ldots, T
$$

where $P_{L}^{t}$ can be calculated using the constant-loss formula [3] given by (7):

$$
P_{L}^{t}=\sum_{i=1}^{N} \sum_{j=1}^{N} P_{i}^{t} B_{i j} P_{j}^{t}+\sum_{i=1}^{N} B_{o i} P_{i}^{t}+B_{o o}
$$

where, $B_{i j}, B_{o i}, B_{o o}$ are the loss parameters also called $B$ coefficients.

- Generating unit RRL

In practice, power generation of each unit $i$ during two consecutive time periods is limited by its RRLs defined by (8) and (9):

$$
\begin{aligned}
& P_{i}^{t-1}-P_{i}^{t} \leq R_{i}^{\text {down }} \\
& P_{i}^{t}-P_{i}^{t-1} \leq R_{i}^{u p}
\end{aligned}
$$

where, $P_{i}^{t-1}$ is the previous output real power of the $i$-th machine. $R_{i}^{\text {down }}$ and $R_{i}^{u p}$ are the down-ramp and up-ramp limits of the $i$-th unit in (MW/time period).

As one of the contributions of the present work, the RRL constraints are taken into account during the transition from the last hour of the current day to the first hour of the next day. Two constraints are embedded in the problem formulation and they are described by (10) and (11):

$$
\begin{aligned}
& P_{i}^{24}-P_{i}^{1} \leq R_{i}^{\text {down }} \\
& P_{i}^{1}-P_{i}^{24} \leq R_{i}^{u p}
\end{aligned}
$$

- $\mathrm{POZ}$ constraints

The POZ constraints are described as:

$$
P_{i}^{t} \in\left\{\begin{array}{l}
P_{i}^{\min } \leq P_{i}^{t} \leq P_{i, 1}^{\text {down }} \\
P_{i, k-1}^{u p} \leq P_{i}^{t} \leq P_{i, k}^{\text {down }} \quad, k=2, \ldots, z_{i} \\
P_{i, z_{i}}^{u p} \leq P_{i}^{t} \leq P_{i}^{\max }
\end{array}\right.
$$

where, $P_{i, k}^{\text {down }}$ and $P_{i, k}^{u p}$ are down and up bounds of POZ number $k, z_{i}$ is the number of POZ for the $i$-th unit due to the vibrations in the shaft or other machine faults. Therefore, the machine has discontinuous input-output characteristics [19]. Figure 2 shows the fuel cost function for a typical thermal unit with POZ constraints.

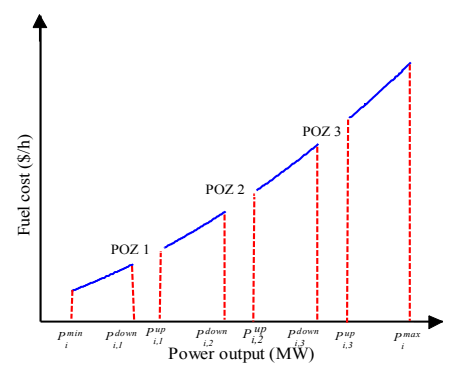

Fig. 2. Cost function for a thermal unit with POZ constraints.

By considering the generation capacity, RRL and POZ constraints, the minimum and maximum limits of the power generation $P_{i}^{t}$ of the $i$-th unit for the period $t$ are modified as:

$$
P_{i}^{t} \in\left\{\begin{array}{l}
\max \left(P_{i}^{\min }, P_{i}^{t-1}-R_{i}^{\text {down }}\right) \leq P_{i}^{t} \\
\quad \leq \min \left(P_{i}^{\max }, P_{i}^{t-1}+R_{i}^{u p}, P_{i, 1}^{\text {down }}\right) \\
\max \left(P_{i}^{\min }, P_{i}^{t-1}-R_{i}^{\text {down }}, P_{i, k-1}^{u p}\right) \leq P_{i}^{t} \\
\quad \leq \min \left(P_{i}^{\max }, P_{i}^{t-1}+R_{i}^{u p}, P_{i, k}^{\text {down }}\right) \\
\max \left(P_{i}^{\min }, P_{i}^{t-1}-R_{i}^{\text {down }}, P_{i, z_{i}}^{u p}\right) \leq P_{i}^{t} \\
\quad \leq \min \left(P_{i}^{\max }, P_{i}^{t-1}+R_{i}^{u p}\right)
\end{array}\right.
$$

where $k=2, \ldots, z_{i}$

\section{ORIGINAL ABC ALGORITHM OVERVIEW}

$\mathrm{ABC}$ algorithm [17] is an efficient and robust technique for several optimization problems. As all swarm-based techniques, $\mathrm{ABC}$ algorithm starts by generating randomly an initial population of $S N$ solutions. Each solution is considered as a food source and it corresponds to an employed bee. $S N$ is half of the entire population size $(P N)$. The onlooker bees constitute the second half. If $D$ is the number of the decision variables, an $i$-th solution $X^{i}$ will be represented by $X^{i}=\left[\begin{array}{llll}x_{1}^{i} & x_{2}^{i} & \ldots & x_{D}^{i}\end{array}\right]$. The fitness function evaluated at the solution $X^{i}$, signifies the nectar quantity of the corresponding food source estimated by an employed bee:

$$
f i t\left(X^{i}\right)=\left\{\begin{array}{l}
\frac{1}{1+f\left(X^{i}\right)}, f\left(X^{i}\right) \geq 0 \\
1+\left|f\left(X^{i}\right)\right|, f\left(X^{i}\right)<0
\end{array}\right.
$$

where, $f\left(X^{i}\right)$ is the objective function estimated at $X^{i}$.

The probability $p_{i}$ to choose the candidate solution $X^{i}$ by an onlooker bee is expressed as follows. 


$$
p_{i}=\frac{f i t\left(X^{i}\right)}{\sum_{n=1}^{S N} f i t\left(X^{n}\right)}
$$

The onlooker bees update the selected food source $X^{i}$ to discover a new one. The new solution $V^{i}$ is generated by modifying only one parameter $x_{j}^{i}$ of $X^{i}$ as:

$$
v_{j}^{i}=x_{j}^{i}+\varphi_{j}^{i}\left(x_{j}^{i}-x_{j}^{k}\right)
$$

where, indices $k$ and $j$ are chosen randomly from $\{1,2, \ldots, S N\}$ and $\{1,2, \ldots, D\}$, respectively. The index $k$ must be different from $i$. The parameter $\varphi_{j}^{i}$ is a real number $\in[0,1]$ generated from the uniform distribution. During the onlooker phase, a greedy selection between food sources $V^{i}$ and $X^{i}$ will be done. If an employed bee's food source cannot be improved through a pre-specified triggering threshold, called LIMIT, it becomes a scout and its solution will be abandoned. If $X^{i}$ is an abandoned solution, the converted scout-bee starts to search for a new solution randomly according to (17):

$$
x_{j}^{i}=X_{j}^{\min }+\operatorname{rand}(0,1)\left(X_{j}^{\max }-X_{j}^{\min }\right)
$$

where, $X_{j}^{\max }$ and $X_{j}^{\min }$ are bounds of the food source in dimension $j$.

\section{PROPOSED OPTIMIZATION ALGORITHM}

An enhanced version of the classical $A B C$ technique is presented to enhance its exploitation and exploration abilities related to the onlooker and scout bees respectively. In [18-19], the $\mathrm{ABC}$ method was criticized for the random selection of the $j$-th dimension because it may slow the convergence of the algorithm and increase the risk of convergence of the search phase to a local optima. To ovoid these limitations, authors in [19] incorporated a new method, called grenade explosion based method (GEM), into the employed bee and onlooker bee phases. The idea behind this technique is to mimic the grenade explosion principle where, the fitness function is the overall damage of the explosion. In each cycle of the proposed method, it is assumed that there is only one grenade with one piece of shrapnel for each decision parameter. A procedure guideline for tuning the parameters of GEM is given in [19]. The shrapnel pieces are thrown in all dimensions to collect information about the area of the explosion which is considered as the old food source as given in (18). Then, a set of new food sources is proposed by the onlooker bees based on the damageper-shrapnel degree. This allows reaching the global solution more quickly. As given in (19), the optimal search dimension (OSD) of the new candidate $V_{O S D}^{i}$ corresponds to the maximum damage in all directions.

$$
v_{t}^{i}=x_{t}^{i}+\varphi_{t}^{i}\left(x_{t}^{i}-x_{t}^{k}\right)
$$

where, $k \in\{1,2, \ldots, S N\}$ is a randomly chosen index and $k \neq i$ and $t \in\{1,2, \ldots, D\} . \varphi_{t}^{i} \in[0,1]$ is a random number.

$$
f i t\left(V_{O S D}^{i}\right)=\max \left\{f i t\left(V_{t}^{i}\right) \mid t=1,2, \ldots, D\right\}
$$

As explained above, a greedy selection between the new solution $V^{i}$ and the old one $X^{i}$ is applied. In order to improve the global and local exploration abilities of the optimization algorithm and ensure the convergence into the global solution within a short calculation time, the Cauchy operator is embedded in the scout bee phase. The incorporation of a Cauchy operator in optimization techniques has been employed in some algorithms to enhance the global search ability [16]. This enhancement is due to the long tail of Cauchy distribution compared to other operators such as Gaussian distribution. As given in Figure 3, the standard Gaussian function has a large probability within the interval $[-3,3]$. Nevertheless, it is possible to make larger jumps in the search space using Cauchy operator.

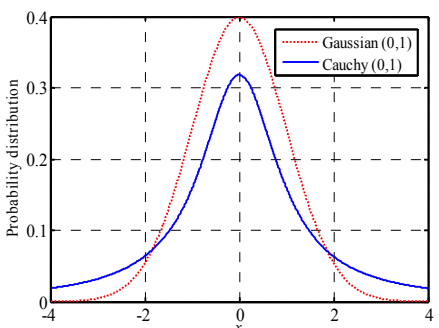

Fig. 3. Standard Cauchy and Gaussian distributions

In this study, the origin-centered Cauchy distribution with unit scale parameter is used. Thus, the new solution provided from an abandoned solution $X^{i}$ will be obtained using (20) instead of (17).

$$
x_{j}^{i}=x_{j}^{i} \operatorname{CAUCHY}(0,1)
$$

where,

$$
\operatorname{CAUCHY}(0,1)=\frac{1}{\pi\left(1+\left(x_{j}^{i}\right)^{2}\right)}
$$

Based on the above explanation, the main steps of the modified $\mathrm{ABC}$ algorithm are listed below.

- Step 1: Initialization

Preset population size $P N(S N)$

Initialize iteration: Iter $=0$

Preset Maximum Cycle Number $(M C N)$

Fix triggering threshold (LIMIT)

Initialize food sources using (4)

- Step 2: Population evaluation

Estimate fitness value of each source using (1)

Initialize failure counter of each source $\mathrm{Xi}, F C(i)=0$

- Step 3: Cycle increment

- Step 4: Employed bees' phase

for $\mathrm{i}=1$ to $S N$ do

Produce new source $V_{O S D}^{i}$ from $X^{i}$ using (19);

Estimate the fitness value of $V_{O S D}^{i}$ using (19); 
Apply greedy selection between $V_{O S D}^{i}$ and $X^{i}$; end for

- Step 5: Onlooker bees' phase

$\mathrm{t}=0 ; \mathrm{i}=1$;

while $(\mathrm{t}<S N)$

ifrand $<\operatorname{Prob}(\mathrm{i})$

$t=t+1$

Apply steps 1 to 2 of employed bee phase;

end if

Apply greedy selection between $V_{O S D}^{i}$ and $X^{i}$;

if solution does not improve

$F C(i)=F C(i)+1$;

end if

$i=i+1$;

end while

- Step 6: Memorization of the best food source

- Step 7: Conditional test 1:

If cycle $<\mathrm{MCN}$ go to step 8 , else go to step 10

- Step 8: Conditional test 2:

if $F C(i)<$ LIMIT go to step 3, else go to step 9

- Step 9: Scout bee phase

Apply Cauchy operator using (20) and (21) and go to step 3

- Step 10: End

\section{EXTENDED ENTROPY-WEIGHTED REFERENCE APPROACH}

The DEED is a bi-objective optimization problem with contradictory functions. Consequently, results with any optimization algorithm will be a set of non-dominated solutions called Pareto front. Providing an adequate candidate Pareto-optimal solution for the decision makers (DM) is a persistent requirement. In the present study, a Shannon's entropy-based multi-attribute decision-making (MADM) method is proposed to rank the obtained non-dominated solutions. The concept of Shannon's entropy is used in several scientific domains such as material selection [22] and singlesensor fault location [24]. This concept can be adopted for MOPs with $\mathrm{n}$ objective functions and $\mathrm{m}$ non-dominated solutions as:

- Step 1: Construct the decision matrix $X=\left(x_{i j}\right)_{m \times n}$, where, $x_{i j}$, called performance index, is the value of the $j$-th function for the $i$-th solution.

- Step 2: Normalize matrix $X$ in order to have performance indices comparable and dimensionless.

$$
x_{i j}^{*}=\frac{x_{i j}}{\sum_{i=1}^{m} x_{i j}^{2}}
$$

- Step 3: Calculate entropy $E_{j}$ as follows.

$$
E_{j}=-E_{0} \sum_{i=1}^{m} x_{i j}^{*} \ln x_{i j}^{*}, j=1, \ldots, n
$$

where $E_{0}=\frac{1}{\ln (m)}$ and $\ln x_{i j}^{*}$ is considered 0 for $x_{i j}^{*}=0$.

- Step 4: Compute the weight of each objective $j$.

$$
w_{j}=\frac{1-E_{j}}{\sum_{j=1}^{n}\left(1-E_{j}\right)}
$$

The decision maker can assign a degree of importance $S_{j}$ for each objective function $j$ called subjective weight. Thus, weights should be modified as follows.

$$
w_{j}^{*}=\frac{s_{j} w_{j}}{\sum_{j=1}^{n} s_{j} w_{j}}
$$

- Step 5: Determine the $i$-th co-ordinate reference point (CRP) per objective function. It is defined as the highest performance index for maximization and the lowest for minimization [22]. However the DEED is a minimization problem. The CRP can be found using (26):

$$
r_{j}=\min _{i} x_{i j}^{*}
$$

- Step 6: Calculate the deviation of each performance index from the CRP for each objective function. Then, determine the maximum deviation for each alternative respecting all objective functions using (27). Each non-dominated solution is considered as alternative.

$$
z_{i}=\max _{j}\left|w_{j}^{*} r_{j}-w_{j}^{*} x_{i j}^{*}\right|
$$

- Step 7: Classify all alternatives according to their maximum deviations then, select the alternative with rank one as the optimal alternative.

\section{IMPLEMENTATION OF THE PROPOSED ALGORITHM}

Having been applied for the first time to solve one of the main power system problems, which is the DEED problem, the GCABC is to be tested in this section on two well-known benchmark power systems. In order to demonstrate the effectiveness of the proposed optimization technique, a comparison with $\mathrm{ABC}$ algorithm and more than six metaheuristic-based techniques used for solving the power dispatch problem is presented. For fair comparison, GCABC and $\mathrm{ABC}$ algorithms have been implemented with the same parameters. Results were obtained using MATLAB R2009a installed on a 64-bit PC with i7-4510U CPU @ 2.60 GHz.

\section{A. Case 1: Six-Unit System}

The six-unit system with quadratic cost and emission functions is used to test the GCABC algorithm in solving the 
SEED problem. The system data are taken from [25]. The Bloss matrix of this system is given below:

$$
B=10^{-6}\left[\begin{array}{cccccc}
140 & 17 & 15 & 19 & 26 & 22 \\
17 & 60 & 13 & 16 & 15 & 20 \\
15 & 13 & 65 & 17 & 24 & 19 \\
19 & 16 & 17 & 71 & 30 & 25 \\
26 & 15 & 24 & 30 & 69 & 32 \\
22 & 20 & 19 & 25 & 32 & 85
\end{array}\right]
$$

Power production cost and emission were optimized simultaneously by minimizing the combined objective function (3). The PPF of the six thermal units are given in Figure 4, hence, the average of these factors is $\lambda=51.3073 \$ /$ ton .

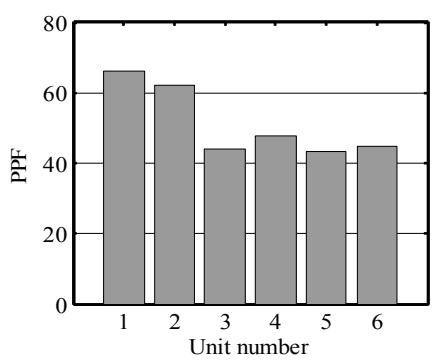

Fig. 4. PPF for the six-unit system

To collect the required number of non-dominated solutions, the proposed optimization algorithm was performed several times. Best, worst, mean solutions and standard deviation (STD) of the 10 trials obtained by the proposed GCABC and the original $\mathrm{ABC}$ algorithms, for total demand power about of $700 \mathrm{MW}$ and the convergence ratio $C_{R}$ that equals to the number of successful trials $N_{S}$ divided by the total number of trials $N_{T}$, are shown in Table I.

$$
C_{R}=\frac{N_{s}}{N_{T}}
$$

TABLE I. RESULTS FOR 10 TRIALS

\begin{tabular}{|c|c|c|c|c|}
\hline & \multicolumn{2}{|c|}{ Total fuel cost (\$/h) } & \multicolumn{2}{c|}{ Total emission (ton/h) } \\
\hline & GCABC & ABC & GCABC & ABC \\
\hline Best & 36912.24 & 36918.56 & 434.1320 & 434.1331 \\
\hline Worst & 36912.63 & 36913.46 & 434.1568 & 434.3861 \\
\hline Mean & 36912.29 & 36913.03 & 434.1368 & 434.2349 \\
\hline STD & 0.1176 & 0.3658 & $0.7328 \mathrm{E}-02$ & $8.3723 \mathrm{E}-02$ \\
\hline CR (\%) & 90 & 70 & 90 & 60 \\
\hline
\end{tabular}

Table I shows the robustness of the convergence of the GCABC and its good stability compared to the original ABC. Pareto fronts for both GCABC and classical ABC algorithms are given in Figure 5. It can be seen that when the total cost in $\$ / \mathrm{h}$ is minimized, the total emission in ton $/ \mathrm{h}$ is at its maximum value and vice versa. Figure 5 shows that Pareto solutions obtained using GCABC are well distributed when compared to those obtained using $\mathrm{ABC}$ algorithm. In addition, GCABC gives the best solutions for production cost or emission. The best compromise solution, shown in Figure 5, has been extracted from the Pareto solutions using the EEWR approach.
The convergence of the proposed algorithm is depicted in Figure 6. GCABC algorithm converges into optimum solution at iteration 80 for best cost (Figure 6(a)) and at iteration number 54 for best emission (Figure 6(b)).

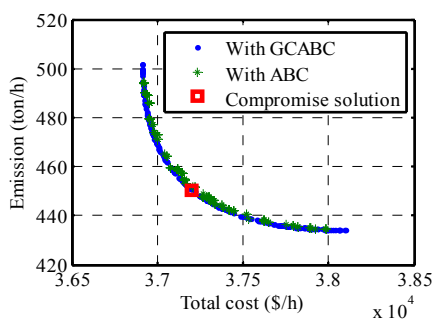

Fig. 5. Pareto solutions

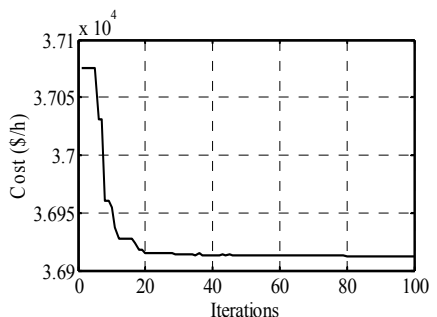

(a)

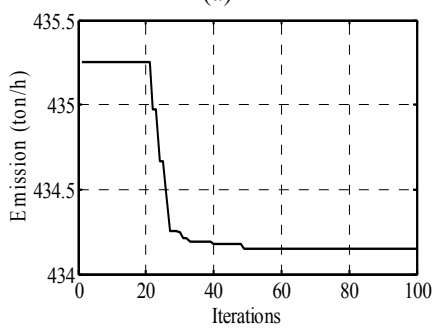

(b)

Fig. 6. Convergence of the proposed algorithm

The optimum power generation of all units for minimum cost and minimum emission using GCABC, ABC and other metaheuristic techniques [25] are summarized in Tables II and III. These Tables show that GCABC, FA (Firefly algorithm), BA (Bat Algorithm) and HYB (Hybrid algorithm) outperform the classical $\mathrm{ABC}$ algorithm. The investigation of the equality constraint described by (7) for each method, shows that GCABC is more accurate than FA, BA and HYB techniques. This is clearly depicted in Figure 7 where, an accuracy value of each method is given by calculating the difference between the total production and the sum of the total load and power losses as described in (29). In Figure 7, the accuracy is calculated with 4 digits after the decimal point.

$$
\text { Accuracy }=\sum_{i=1}^{N} P_{i}-\left(P_{D}+P_{L}\right)
$$

\section{B. Case 2: DEED Problem for the Ten-Unit System with POZs}

The well-known benchmark test system, called the ten-unit system, was used to prove the feasibility of GCABC for solving the DEED problem including all operating constraints 
such as, VPLE, RRL and POZ. The RRLs corresponding to the transition from the last hour of the current day to the first hour of the next day, given by (10) and (11) were taken into account.

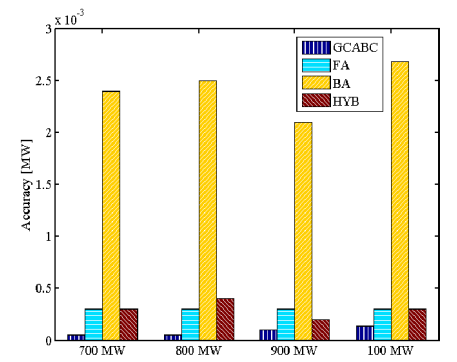

Fig. 7. Accuracy of GCABC, FA, BA and HYB for different load

TABLE II. BEST SOLUTION FOR MINIMUM COST (PD=700MW)

\begin{tabular}{|c|c|c|c|c|c|}
\hline Method & GCABC & ABC & FA & BA & HYB \\
\hline P1 (MW) & 281,562 & 318,442 & 283,151 & 282,862 & 282,491 \\
\hline P2 (MW) & 100,000 & 100,000 & 100,000 & 100,000 & 100,000 \\
\hline P3 (MW) & $1,192.695$ & $1,270.720$ & $1,188.216$ & $1,189.333$ & $1,188.833$ \\
\hline P4 (MW) & $1,193.578$ & $1,201.647$ & $1,188.329$ & $1,186.760$ & $1,185.778$ \\
\hline P5 (MW) & $2,294.322$ & $2,269.768$ & $2,309.801$ & $2,307.614$ & $2,305.090$ \\
\hline P6 (MW) & $2,132.045$ & $2,030.748$ & $2,124.811$ & $2,127.731$ & $2,132.178$ \\
\hline Losses (MW) & 194.202 & 191.325 & 194.311 & 194.324 & 194.373 \\
\hline CT (\$/h) & 36912.24 & 36918.56 & 36912.19 & 36912.08 & 36912.19 \\
\hline ET(ton/h) & 500.53 & 494.25 & 501.02 & 501.02 & 501.08 \\
\hline
\end{tabular}

TABLE III. BEST SOLUTION FOR MINIMUM EMISSION ( $\mathrm{PD}=700 \mathrm{MW})$

\begin{tabular}{|c|c|c|c|c|c|}
\hline Method & GCABC & ABC & FA & BA & HYB \\
\hline P1 (MW) & 804.827 & 767.280 & 801.523 & 801.431 & 801.506 \\
\hline P2 (MW) & 822.259 & 797.532 & 824.019 & 824.033 & 824.054 \\
\hline P3 (MW) & $1,140.623$ & $1,097.203$ & $1,139.655$ & $1,139.684$ & $1,139.570$ \\
\hline P4 (MW) & $1,137.692$ & $1,182.489$ & $1,134.758$ & $1,134.763$ & $1,134.851$ \\
\hline P5 (MW) & $1,631.220$ & $1,695.130$ & $1,634.493$ & $1,634.530$ & $1,634.436$ \\
\hline P6 (MW) & $1,628.759$ & $1,626.760$ & $1,630.944$ & $1,630.950$ & $1,630.975$ \\
\hline Losses (MW) & 165.378 & 166.394 & 165.398 & 165.397 & 165.397 \\
\hline CT (\$/h) & 38105.00 & 37987.80 & 38101.09 & 38100.95 & 38101.13 \\
\hline ET(ton/h) & 434.13 & 434.66 & 434.13 & 434.13 & 434.13 \\
\hline
\end{tabular}

The problem becomes highly non-linear and more complicated. The B-loss matrix of the system is given as:

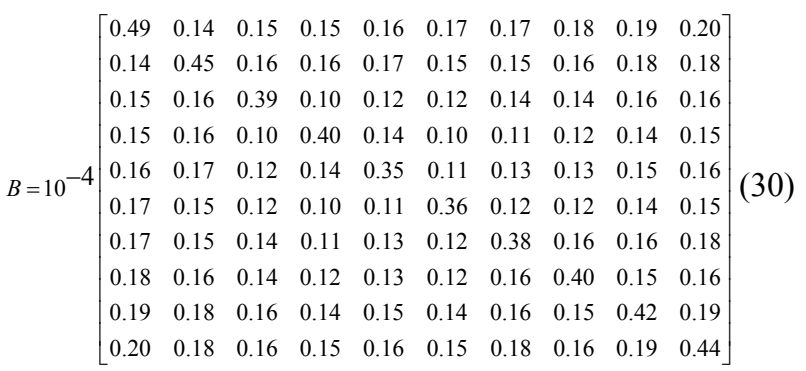

Total cost and emission functions were minimized individually and simultaneously according to the variation of the power demand $P_{D}^{t}$ in MW over a time-period of one day, subdivided into 24 time intervals of one hour (relevant data were taken from [12]). Results obtained using GCABC were compared with other techniques used recently in this field such as IBFA [23] and NSGAII [12]. The comparison results, shown in Table IV, confirm that the proposed GCABC outperforms the other optimization techniques in providing the optimum generation schedule for the DEED problem. This good performance was obtained despite the constraints (10) and (11), which were considered in GCABC.

TABLE IV. META-HEURISTIC TECHNIQUES COMPARISON

\begin{tabular}{|c|c|c|c|c|}
\hline & \multicolumn{2}{|c|}{ Minimum total cost (\$/h) } & $\begin{array}{c}\text { Minimum total emission } \\
\text { (ton/h) }\end{array}$ \\
\hline Method & $\begin{array}{c}\text { Without } \\
\text { POZs }\end{array}$ & With POZs & $\begin{array}{c}\text { Without } \\
\text { POZs }\end{array}$ & $\begin{array}{c}\text { With } \\
\text { POZs }\end{array}$ \\
\hline GCABC & 2474472.8 & 2484750.6 & 293416.3 & 293651.3 \\
\hline IBFA & 2481733.3 & NA & 295833.0 & NA \\
\hline NSGAII & $2.5168 \times 106$ & NA & $3.1740 \times 105$ & NA \\
\hline
\end{tabular}

\section{CONCLUSION}

Dynamic economic emission dispatch (DEED) is a tricky optimization problem. The quality of its optimal solution is influenced by the operating constraints, such as valve-point loading effects, prohibited operating zones and ramp rate limits (RRLs). Within this context, the present study proposed a new artificial bee colony (ABC)-based technique for solving the DEED problem. Moreover, power balance constraint was also considered. Unlike previous works, the RRLs were embedded in the solution procedure during the transition from one day to the next. The proposed optimization technique incorporates the grenade explosion method and Cauchy operator in the classical $\mathrm{ABC}$ algorithm to avoid the random search in the different $\mathrm{ABC}$ phases. To provide adequate compromise solution for the decision makers, an approach based on extended entropyweighted reference was proposed. The validation of the proposed optimization algorithm was verified on ten-unit system test with POZs. Comparison results with more than ten metaheuristic techniques show that the proposed algorithm gives the best optimum solutions.

\section{REFERENCES}

[1] P. K. Roy, S. A. Bhui, "Multi-objective hybrid evolutionary algorithm for dynamic economic emission load dispatch", International Transactions on Electrical Energy Systems, Vol. 26, No. 1, pp. 49-78, 2016

[2] R. Muthuswamy, M. Krishnan, K. Subramanian, B. Subramanian, "Environmental and economic power dispatch of thermal generators using modified NSGA-II algorithm", International Transactions on Electrical Energy Systems, Vol. 25, No. 8, pp. 1552-1569, 2014

[3] K. Tlijani, T. Guesmi, H. Hadj Abdallah, "Dynamic coupled ActiveReactive Dispatch Including SVC Devices with Limited Switching Operations", Arabian Journal for Science and Engineering, Vol. 42, No. 7, pp. 2651-2661, 2017

[4] X. Jiang, J. Zhou, H. Wang, Y. Zhang, "Dynamic environmental economic dispatch using multiobjective differential evolution algorithm with expanded double selection and adaptive random restart", International Journal of Electrical Power \& Energy Systems, Vol. 49, pp. 399-407, 2013

[5] K. Vaisakh, P. Praveena, K. Naga Sujatha, "Solution of dynamic economic emission dispatch problem by hybrid bacterial foraging algorithm", International Journal of Computer Science and Electronics Engineering, Vol. 2, No. 1, pp. 58-64, 2014

[6] Z. Zhu, J. Wang, M. H. Baloch, "Dynamic economic emission dispatch using modified NSGA-II", International Transactions on Electrical Energy Systems, Vol. 26, No. 12, pp. 2684-2698, 2016 
[7] M. K. Sharma, P. Phonrattanasak, N. Leeprechanon, "Improved bees algorithm for dynamic economic dispatch considering prohibited operating zones", IEEE-Innovative Smart Grid Technologies-Asia (ISGT ASIA), Bangkok, Thailand, November 3-6, 2015

[8] M. I. Behnam, R. Abbas, S. Alireza, "Nonconvex dynamic economic power dispatch problems solution using hybrid immune-genetic algorithm", IEEE Systems Journal, Vol. 7, No. 4, pp. 777-785, 2013

[9] T. Sen, H. D. Mathur, "A new approach to solve economic dispatch problem using a hybrid ACO-ABC-HS optimization algorithm", International Journal of Electrical Power \& Energy Systems, Vol. 78, pp. 735-744, 2016

[10] Z. Liang, J. D. Glover, "A zoom feature for a dynamic programming solution to economic dispatch including transmission losses", IEEE Transactions on Power Systems, Vol. 7, No. 2, pp. 544-550, 1992

[11] M. C. W. Gar, J. G. Aganagic, B. Tony Meding Jose, S. Reeves, "Experience with mixed integer linear programming based approach on short term hydrothermal scheduling", IEEE Transactions on Power Systems, Vol. 16, No. 4, pp. 743-749, 2001

[12] M. Basu, "Dynamic economic emission dispatch using nondominated sorting genetic algorithm-II”, International Journal of Electrical Power \& Energy Systems, Vol. 30, pp. 140-149, 2008

[13] J. B. Park, K. S. Lee, J. R. Shin, K. Y. Lee, "A particle swarm optimization for economic dispatch with nonsmooth cost functions", IEEE Transactions on Power Systems, Vol. 20, No. 1, pp. 34-42, 2005

[14] I. Ziane, F. Benhamida, A. Graa, "Simulated annealing algorithm for combined economic and emission power dispatch using max/max price penalty factor", Neural Computing and Applications, Vol. 28 (Suppl. 1), pp. 197-205, 2017

[15] W. M. Lin, F. S. Cheng, M. T. Tsay, "An improved Tabu search for economic dispatch with multiple minima", IEEE Transactions on Power Systems, Vol. 17, No. 1, pp. 108-112, 2002

[16] J. G. Zheng, C. Q. Zhang, Y. Q. Zhou, "Artificial bee colony algorithm combined with grenade explosion method and Cauchy operator for global optimization", Mathematical Problems in Engineering, Vol. 2015, Article ID 739437, 2015

[17] D. Karaboga, An idea based on honey bee swarm for numerical optimization, Tech Rep TR06, Erciyes University, Engineering Faculty, Computer Engineering Department, Turkey, 2005

[18] C. Zhang, J. Zheng, Y. Zhou, "Two modified artificial bee colony algorithms inspired by grenade explosion method", Neurocomputing, Vol. 151, No. 3, pp. 1198-1207, 2015

[19] A. Ahrari, A. A. Atai, "Grenade explosion method-A novel tool for optimization of multimodal functions", Applied Soft Computing, Vol. 10, pp. 1132-1140, 2010

[20] R. Venkata Rao, "A material selection model using graph theory and matrix approach", Material Science Engineering: A, Vol. 431, No. 1-2, pp. $248-255,2006$

[21] M. Soleimani-Damaneh, M. Zarepisheh, "Shannon's entropy for combining the efficiency results of different DEA models: Method and application", Expert Systems with Applications, Vol. 36, pp. 5146-5150, 2009

[22] A. Hafezalkotob, A. Hafezalkotob, "Extended MULTIMOORA method based on Shannon entropy weight for materials selection", Journal of Industrial Engineering International, Vol. 12, No. 1, pp. 1-13, 2016

[23] N. Pandit, A. Tripathi, S. Tapaswi, M. Pandit, "An improved bacterial foraging algorithm for combined static/dynamic", Applied Soft Computing, Vol. 12, No. 11, pp. 3500-3513, 2012

[24] A. Hafezalkotob, A. Hafezalkotob, "Fuzzy entropy-weighted MULTIMOORA method for materials selection", Journal of Intelligent \& Fuzzy Systems, Vol. 31, No. 3, pp. 1211-1226, 2016

[25] Y. A. Gherbi, H. Bouzeboudja, F. Z. Gherbi, "The combined economic environmental dispatch using new hybrid metaheuristic", Energy, Vol. 115, Part 1, pp. 486-477, 2016 